\title{
Approximation polynomiale et extension holomorphe avec croissance sur une variété algébrique
}

\author{
par A. ZERIAHI (Toulouse)
}

\begin{abstract}
We first give a general growth version of the theorem of Bernstein-WalshSiciak concerning the rate of convergence of the best polynomial approximation of holomorphic functions on a polynomially convex compact subset of an affine algebraic manifold. This can be considered as a quantitative version of the well known approximation theorem of Oka-Weil. Then we give two applications of this theorem. The first one is a generalization to several variables of Winiarski's theorem relating the growth of an entire function to the rate of convergence of its best polynomial approximation; the second application concerns the extension with growth of an entire function from an algebraic submanifold to the whole space.
\end{abstract}

Introduction. Soit $K$ un compact polynomialement convexe de $\mathbb{C}^{n}$. D'après le théorème d'Oka-Weil, toute fonction $f$ holomorphe sur un voisinage de $K$ peut être approchée uniformément sur $K$ par des polynômes. Le théorème de Bernstein-Walsh-Siciak ([Si,2]) est une version quantitative précise du théorème d'Oka-Weil qui exprime le degré de convergence de la meilleure approximation polynomiale de $f$ sur le compact $K$ à l'aide du niveau, par rapport à la fonction extrémale de Siciak de $K$, du plus grand domaine sur lequel $f$ est holomorphe, pourvu que $K$ soit régulier.

Nous donnons ici une version générale à croissance du théorème de Bernstein-Walsh-Siciak qui généralise notre résultat antérieur dans deux directions. Tout d'abord, le théorème d'approximation que nous obtenons (théorème 3.2) s'applique aux fonctions holomorphes sur une variété algébrique affine lisse quelconque, ensuite l'approximation polynomiale a lieu sur un compact non pluripolaire quelconque de cette variété.

Nous donnons enfin deux applications du théorème obtenu. La première est une généralisation du théorème classique de Winiarski ([Wi,1]) qui relie la rapidité de la meilleure approximation polynomiale d'une fonction entière

1991 Mathematics Subject Classification: 32A15, 32A22, 32D15, 32E30.

Key words and phrases: Green function, $L^{2}$ estimation, approximation, growth, extension. 
sur le compact $K$ à son type de croissance sur les domaines de niveau de la fonction extrémale de $K$ (théorème 4.1 ).

La deuxième application concerne le prolongement d'une fonction entière définie sur une sous-variété algébrique lisse de $\mathbb{C}^{n}$ en une fonction entière sur $\mathbb{C}^{n}$ avec un contrôle précis du type de croissance (théorème 5.1).

1. Estimations $L^{2}$ sur une variété algébrique affine. Nous allons rappeler dans ce paragraphe le théorème fondamental d'existence de Hörmander-Nakano-Skoda. Nous aurions pu renvoyer à Skoda ([Sk]) et Demailly ([De]) pour les résultats dont nous aurons besoin, mais il nous a semblé commode de les rappeler ici, ne serait ce que pour fixer les notations.

Soit $X$ une sous-variété algébrique de $\mathbb{C}^{\nu}$ de dimension pure $n$ muni de la métrique Kählérienne induite par la métrique plate de $\mathbb{C}^{\nu}$. La forme de Kähler associée est

$$
\beta=\frac{1}{4} d d^{c} \tau=\frac{i}{2} \partial \bar{\partial} \tau
$$

où $\tau(x)=|x|^{2}$ sur $X,|\cdot|$ étant la norme hermitienne sur $\mathbb{C}^{\nu}$.

La courbure de la variété Kählérienne $(X, \beta)$, appelée courbure de Ricci de la métrique $\beta$ et notée $\operatorname{Ricci}(\beta)$, peut être définie par

$$
\operatorname{Ricci}(\beta)=-i c\left(K_{X}\right) \text {, }
$$

où $c\left(K_{X}\right)$ est la $(1,1)$-forme de courbure du fibré canonique $K_{X}=\bigwedge^{n} T^{*} X$. Dans un repère local de $K_{X}$, la forme de courbure s'exprime de la façon suivante :

Soit $\left(U, z_{1}, \ldots, z_{n}\right)$ un système de coordonnées locales de $X$, de sorte que $d z_{1} \wedge \ldots \wedge d z_{n}$ soit un repère local de $K_{X}$ au-dessus de $U$. Alors il existe une fonction $h>0$ sur $U$ telle que

$$
i^{n^{2}} d z_{1} \wedge \ldots \wedge d z_{n} \wedge d \bar{z}_{1} \wedge \ldots \wedge d \bar{z}_{n}=h^{2} \beta_{n},
$$

où $\beta_{n}=(1 / n !) \beta^{n}$ est la forme volume sur $X$. On a alors $c\left(K_{X}\right)=\bar{\partial} \partial \log h$ sur $U$.

Le théorème d'existence s'énonce ainsi :

ThÉORÈme $([\mathrm{Sk}])$. Soit $\varphi \in L_{\mathrm{loc}}^{1}(X)$ telle que la condition suivante soit vérifiée :

$$
\frac{1}{2} d d^{c} \varphi+\operatorname{Ricci}(\beta) \geq \lambda \beta^{n},
$$

où $\lambda$ est une fonction continue et positive sur $X$. Alors pour toute $(0,1)$ forme $g \in L_{(0,1)}^{2}(X$, loc $)$ vérifiant $\bar{\partial} g=0$, il existe $u \in L_{\mathrm{loc}}^{2}(X)$ telle que $\bar{\partial} u=g$ et

$$
\int_{X}|u|^{2} e^{-\varphi} \beta_{n} \leq \int_{X} \lambda^{-1}|g|^{2} e^{-\varphi} \beta_{n}
$$

pourvu que le second membre soit fini. 
Pour réaliser la condition de courbure (1.1) il suffit en général de trouver une fonction $\psi$ p.s.h. sur $X$ telle que

$$
\frac{1}{2} d d^{c} \psi+\operatorname{Ricci}(\beta) \geq 0 .
$$

En posant alors

$$
\varphi=\psi+2 \log (1+\tau)
$$

la condition (1.1) est réalisée avec la fonction

$$
\lambda=(1+\tau)^{-2} \text {. }
$$

Pour déterminer $\psi$ dans le cas qui nous intéresse, rappelons les calculs faits par Demailly $([\mathrm{De}])$ :

Soit $P_{1}, \ldots, P_{m}$ un système de générateurs de l'idéal $I(X)$ de la variété algébrique $X$ et $q=\nu-n=\operatorname{codim} X$. Pour tous les multi-indices croissants $\alpha=\left(\alpha_{1}, \ldots, \alpha_{q}\right)$ avec $\alpha_{1}, \ldots, \alpha_{q} \in\{1, \ldots, m\}, \beta=\left(\beta_{1}, \ldots, \beta_{q}\right)$ avec $\beta_{1}, \ldots, \beta_{q} \in\{1, \ldots, \nu\}$, on pose

$$
J_{\alpha \beta}(x)=\operatorname{det}\left(\frac{\partial P_{\alpha_{j}}(x)}{\partial z_{\beta_{k}}}\right) .
$$

Soit $U_{\alpha}=\left\{x \in X: \sum_{\beta}\left|J_{\alpha \beta}(x)\right|^{2}>0\right\}$. Alors un calcul élémentaire montre que

$$
\operatorname{Ricci}(\beta)=-\frac{1}{2} d d^{c} \log \left(\sum_{\beta}\left|J_{\alpha \beta}(x)\right|^{2}\right) \quad \text { sur } U_{\alpha} .
$$

Il en résulte facilement que la fonction p.s.h. définie par

$$
\psi(x)=\log \left(\sum_{\alpha, \beta}\left|J_{\alpha \beta}(x)\right|^{2}\right), \quad x \in X,
$$

vérifie la condition (1.3) puisque $X=\bigcup_{\alpha} U_{\alpha}$. Si $1+d_{1}$ est le degré maximum des polynômes $P_{1}, \ldots, P_{m}$, on en déduit que

$$
\psi \leq d_{1} \log (1+\tau)+c_{1} \quad \operatorname{sur} X,
$$

où $c_{1}$ est une constante absolue.

Puisque $X$ est non singulière, les polynômes $P_{1}, \ldots, P_{m}, J_{\alpha \beta}$ n'ont pas de zéros communs sur $X$. Il résulte alors du Nullstellensatz de Hilbert qu'il existe un entier $d_{2} \geq 0$ et une constante $c_{2}$ tels que

$$
\psi \geq-d_{2} \log (1+\tau)+c_{2} \quad \text { sur } X
$$

Notons que si $X=\mathbb{C}^{n}$, il suffit de prendre $\psi \equiv 0$ et dans ce cas $d_{1}=d_{2}=$ 0 et le théorème précédent se réduit au théorème d'existence de Hörmander ([H], thm. 4.4.2).

2. Fonction de Green pluricomplexe sur une variété algébrique affine. Rappelons tout d'abord la définition de la fonction extrémale de 
Siciak-Zakharyuta ([Si,1], [Si,2], [Za]), que nous appellerons fonction de Green pluricomplexe à pôle à l'infini.

Si $E$ est un sous-ensemble borné de $\mathbb{C}^{\nu}$, on définit sa fonction extrémale associée sur $\mathbb{C}^{\nu}$ par la formule

$$
L_{E}(z):=\sup \left\{V(z): V \in \mathcal{L}\left(\mathbb{C}^{\nu}\right), V \mid E \leq 0\right\}, \quad z \in \mathbb{C}^{\nu},
$$

où $\mathcal{L}\left(\mathbb{C}^{\nu}\right)$ est la classe des fonctions plurisousharmoniques $V$ sur $\mathbb{C}^{\nu}$ vérifiant

$$
\sup \left\{V(z)-\log (1+|z|): z \in \mathbb{C}^{\nu}\right\}<\infty .
$$

Soit $X$ une sous-variété algébrique (non-singulière) de $\mathbb{C}^{\nu}$ de dimension pure $n$. On posera $\tau(x)=|x|^{2}$ pour $x \in X$, où $|\cdot|$ est la norme hermitienne sur $\mathbb{C}^{\nu}$. On désignera par $\mathcal{L}(X)$ la classe des fonctions $v$ plurisousharmoniques sur $X$ vérifiant l'estimation

$$
v(x) \leq c_{v}+\frac{1}{2} \log (1+\tau(x)), \quad \forall x \in X .
$$

Pour une partie $E \Subset X$, on pose

$$
l_{E}(x):=\sup \{v(x): v \in \mathcal{L}(X), v \leq 0 \text { sur } E\}, \quad x \in X .
$$

On montre que si $E$ est localement non pluripolaire dans $X$ la régularisée s.c.s. $l_{E}^{*}$ est plurisousharmonique sur $X$ et vérifie l'équation de MongeAmpère complexe ([Sa], $[\mathrm{Ze}, 2])$

$$
\left(d d^{c} l_{E}^{*}\right)^{n}=0 \quad \text { sur } X \backslash \bar{E} .
$$

On montre également $([\mathrm{Ze}, 2])$ que si $E$ est compact et $l_{E}^{*}=0$ sur $E$, alors $l_{E}$ est continue sur $X$. On dit dans ce cas que $E$ est régulier.

On montre aussi que si $K$ est un compact de $X$ alors $L_{K} \mid X=l_{K}$ (voir [Sa], [Ze,2]).

Nous aurons également besoin de la "version uniforme" suivante du théorème d'approximation de Bernstein-Walsh.

Rappelons tout d'abord quelques notations :

Soit $I=I(X)$ l'idéal polynomial de la variété algébrique $X$ et $A(X)$ l'algèbre des fonctions régulières sur $X$ qui s'identifie à $\mathbb{C}\left[z_{1}, \ldots, z_{\nu}\right] / I$. Pour chaque entier $p \geq 1$, on note $A_{p}(X)$ l'ensemble des fonctions régulières $f$ sur $X$ telle que $\sup _{x \in X}\left\{(1+\tau(x))^{-p}|f(x)|^{2}\right\}<\infty$.

Il est clair que si $P \in A_{m}(X)$, on a $m^{-1} \log |P| \in \mathcal{L}(X)$. De la définition de $l_{K}$ (voir (2.2)), il résulte l'inégalité suivante (dite de Bernstein-Walsh) :

(B.W.) $\quad|P(x)| \leq\|P\|_{K} \exp \left(m l_{K}(x)\right), \quad \forall P \in A_{m}(X), \forall x \in X, \forall m \in \mathbb{N}$.

Pour étudier l'approximation polynomiale des fonctions holomorphes sur $X$, on définit pour un compact $K \subset X$ et $f \in \mathcal{C}(K)$,

$$
\varepsilon_{p}(f ; K):=\inf \left\{\|f-P\|_{K}: P \in A_{p}(X)\right\}, \quad p \in \mathbb{N} .
$$

On posera $\Omega_{r}=\Omega(K ; r)$, où

$$
\Omega(K ; r):=\left\{x \in X: l_{K}^{*}(x)<\log r\right\}, \quad r>1 .
$$


ThÉORÈME 2.1 ([Ze,2]). Soit $K$ un compact localement non pluripolaire de $X$. Alors pour tout $r>r_{0}:=\sup _{K} \exp l_{K}^{*}$ et $\theta>1$, il existe une constante $c(\theta, r)>0$ telle que

$$
\varepsilon_{p}(f ; K) \leq c(\theta, r) r^{-p}\|f\|_{\bar{\Omega}_{r \theta}}, \quad \forall p \in \mathbb{N}, \forall f \in \mathcal{O}\left(\bar{\Omega}_{r \theta}\right) .
$$

Ici $\mathcal{O}(E)$ désignera l'espace des fonctions (resp. germes de fonctions) holomorphes sur $E$ lorsque $E$ est ouvert (resp. compact).

On en déduit aisément le corollaire suivant qui sera utile :

Corollaire 2.2. Soit $K$ un compact localement non pluripolaire de $X$. Alors pour tout $r_{2}>r_{1}>r_{0}$, il existe une constante $c\left(r_{1}, r_{2}\right)>0$ telle que

$$
\varepsilon_{p}(f ; K) \leq c\left(r_{1}, r_{2}\right) r_{1}^{-p} \varepsilon_{p}\left(f ; \bar{\Omega}_{r_{2}}\right), \quad \forall p \geq 1, \forall f \in \mathcal{O}\left(\bar{\Omega}_{r_{2}}\right) .
$$

3. Une version à croissance du théorème de Bernstein-Walsh. Dans toute la suite $X$ sera une sous-variété algébrique non singulière de $\mathbb{C}^{\nu}$, de dimension pure $n$.

Soit $V: X \rightarrow]-\infty, \infty[$ une fonction plurisousharmonique vérifiant la condition de croissance suivante :

$$
-\gamma+\frac{1}{2} \log (1+\tau) \leq V \leq \gamma+\frac{1}{2} \log (1+\tau),
$$

où $\gamma>0$ est une constante ne dépendant que de $V$. Posons

$$
\begin{aligned}
& D_{r}=D(V ; r):=\{x \in X: V(x)<\log r\}, \quad r>0, \\
& r_{0}:=\inf \left\{r: D_{r} \neq \emptyset\right\} .
\end{aligned}
$$

On a alors le théorème d'approximation suivant :

ThÉORÈme 3.1. Soit $\theta>1$. Alors il existe une constante $c(\theta)>0$ telle que pour tout $r>r_{0}$ et $f \in \mathcal{O}\left(\bar{D}_{r \theta}\right)$, il existe une suite $\left(Q_{l}\right)_{l \geq 1}$ de fonctions holomorphes sur $X$ vérifiant les deux propriétés suivantes :

$$
\begin{gathered}
\int_{X}\left|Q_{l}\right|^{2}(1+\tau)^{-l-d_{1}-2} \beta_{n}<\infty, \quad \forall l \in \mathbb{N}, \\
\int_{D_{s}}\left|f-Q_{l}\right|^{2}(1+\tau)^{-d_{1}-2} \beta_{n} \leq c(\theta)^{2}(s / r)^{2 l}\left(1+r^{2}\right)^{d_{2}+n-1}\|f\|_{\bar{D}_{r \theta}}^{2},
\end{gathered}
$$

pour tout $l \in \mathbb{N}$ et $\left.s \in] r_{0}, r\right]$ ( $d_{1}$ et $d_{2}$ étant les entiers intervenant dans les conditions (1.7) et (1.8) respectivement).

Démonstration. Soit $r>1, \theta>1$ et $\chi_{r, \theta}$ une fonction telle que $\chi_{r, \theta} \in L_{\mathrm{loc}}^{2}(X), \bar{\partial} \chi_{r, \theta} \in L_{0,1}^{2}(X$, loc $)$ vérifiant $\chi_{r, \theta} \equiv 1$ sur $D_{r}$ et à support dans $D_{r \theta}$, que nous préciserons plus tard.

Soit $f \in \mathcal{O}\left(\bar{D}_{r \theta}\right)$. On va chercher une fonction $Q_{l}$ sous la forme

$$
Q_{l}=f \chi_{r, \theta}-u_{l},
$$


où $u_{l} \in L_{\text {loc }}^{2}(X)$ est à déterminer de telle sorte que la fonction $Q_{l}$ soit holomorphe sur $X$ et vérifie les conditions voulues. On est donc ramené à la résolution de l'équation de Cauchy-Riemann suivante :

$$
\bar{\partial} u_{l}=\bar{\partial}\left(f \chi_{r, \theta}\right)=f \bar{\partial} \chi_{r, \theta} \quad \text { sur } X,
$$

avec un contrôle de la croissance de $u_{l}$.

Nous allons appliquer le théorème de Hörmander-Nakano-Skoda (voir $\S 1$ ).

Pour chaque entier $l$, posons $\varphi_{l}:=2 l V+\psi+2 \log (1+\tau)$, où $\psi$ est la fonction définie par (1.6) et qui réalise la condition de positivité $\frac{1}{2} d d^{c} \psi+$ $\operatorname{Ricci}(\beta) \geq 0$. Alors $\varphi_{l}$ est p.s.h. sur $X$ et vérifie la condition de courbure (1.1) avec $\lambda=(1+\tau)^{-2}$. Il existe donc une fonction $u_{l} \in L_{\mathrm{loc}}^{2}(X)$ solution de l'équation (3.7) avec l'estimation

$$
\int_{X}\left|u_{l}\right|^{2}(1+\tau)^{-2} e^{-2 l V-\psi} \beta_{n} \leq \int_{X}|f|^{2}\left|\bar{\partial} \chi_{r, \theta}\right|^{2} e^{-2 l V-\psi} \beta_{n} .
$$

Comme $\chi_{r, \theta}$ est à support dans $D_{r \theta} \Subset X$, le second membre de (3.8) est fini en vertu des hypothèses faites sur la fonction $\chi_{r, \theta}$. D'autre part, $\chi_{r, \theta} \equiv 1$ sur $D_{r}$ et $V \geq \log r$ sur $X \backslash D_{r}$. Il résulte alors de (3.8) et de la minoration (1.8) sur la fonction $\psi$ que l'on a

$$
\int_{X}\left|u_{l}\right|^{2}(1+\tau)^{-2} e^{-2 l V-\psi} \beta_{n} \leq c(r, \theta)^{2} r^{-2 l}\left(1+r^{2}\right)^{d_{2}}\|f\|_{D_{r \theta}}^{2},
$$

où $c(r, \theta)$ est la constante définie par la formule suivante :

$$
c(r, \theta)^{2}:=\int_{D_{r \theta}}\left|\bar{\partial} \chi_{r, \theta}\right|^{2} \beta_{n} .
$$

Posons $Q_{l}=f \chi_{r, \theta}-u_{l}$ pour $l \in \mathbb{N}$. Alors puisque $u_{l}$ est solution de l'équation (3.7), $Q_{l}$ est holomorphe sur $X$ et puisque $\chi_{r, \theta} \equiv 1$ sur $D_{r}$, on a $u_{l}=f-Q_{l}$. Il en résulte d'après (3.9) que si $s \leq r$, on a

$$
\int_{X}\left|f-Q_{l}\right|^{2}(1+\tau)^{-2} e^{-2 l V-\psi} \beta_{n} \leq c(r, \theta)^{2} r^{-2 l}\left(1+r^{2}\right)^{d_{2}}\|f\|_{\bar{D}_{r \theta}}^{2}
$$

pour tout $l \in \mathbb{N}$. Comme $V \leq \log s$ sur $D_{s}$ et que $\psi \leq d_{1} \log (1+\tau)+c_{1}$ (voir (1.7)), l'estimation (3.11) s'écrit

$$
\int_{D_{s}}\left|f-Q_{l}\right|^{2}(1+\tau)^{-d_{1}-2} \beta_{n} \leq c(r, \theta)^{2}(s / r)^{2 l}\left(1+r^{2}\right)^{d_{2}}\|f\|_{D_{r \theta}}^{2} .
$$

D'après (3.9) et la majoration sur $\psi$, on obtient l'inégalité (3.4) du théorème.

Pour démontrer (3.5), il s'agit de préciser la fonction $\chi_{r, \theta}$ et d'en déduire une majoration convenable de la constante (3.10). Pour ce faire, posons $W=e^{V}$ et soit $\chi_{\theta}$ une fonction de classe $C^{\infty}$ sur $\mathbb{R}$ telle que $\chi_{\theta} \equiv 1$ sur 
$[0,1]$ et $\chi_{\theta} \equiv 0$ sur $[\theta, \infty[$. Considérons la fonction définie par

$$
\chi_{r, \theta}(x)=\chi_{\theta}(W(x) / r), \quad x \in X .
$$

Alors $\bar{\partial} \chi_{r, \theta}=\chi_{\theta}^{\prime}(W / r) \bar{\partial} W / r$ sur $X$. Comme $W \geq 0, W^{2}$ est p.s.h. sur $X$ et $2 d W \wedge d^{c} W \leq d d^{c} W^{2}$ au sens des courants sur $X$. Il en résulte que $|\bar{\partial} W|^{2}$ est localement intégrale sur $X$ et vérifie

$$
\int_{B_{R}}|\bar{\partial} W|^{2} \beta_{n} \leq \int_{B_{R}} d d^{c} W^{2} \wedge \beta_{n-1},
$$

où $B_{R}:=\left\{x \in X: \tau(x)<R^{2}\right\}, R>0$. Un calcul simple montre que pour tout $\theta>1$, il existe une constante $c_{1}>0$ telle que

$$
\int_{B_{R}} d d^{c} W^{2} \wedge \beta_{n-1} \leq c_{1}\left(\sup _{B_{R \theta}} W^{2}\right) R^{-2} \int_{B_{R}} \beta_{n}, \quad \forall R>0 .
$$

D'après une inégalité classique, $X$ étant algébrique, on a $\int_{B_{R} \theta} \beta_{n} \leq c_{2}(R \theta)^{2 n}$, où $c_{2}$ est une constante ne dépendant que de $X$. Il en résulte compte tenu de (3.1), (3.15) et (3.14) qu'il existe une constante $c(\theta)>0$ telle que

$$
\int_{B_{R}}|\bar{\partial} W|^{2} \beta_{n} \leq c(\theta)^{2} R^{2 n}, \quad \forall R>0 .
$$

D'après (3.1) il existe une constante $\delta>0$ telle que $D_{R} \subset B_{R \delta}$. On en déduit compte tenu de (3.16) l'inégalité suivante :

$$
\int_{D_{r \theta}}|\bar{\partial} W|^{2} \beta_{n} \leq c(\theta)^{2} r^{2 n}, \quad \forall r>r_{0} .
$$

Il en résulte alors que $c(r, \theta)^{2} \leq c(\theta)^{2} r^{2 n-2}$; ce qui, compte tenu de (3.12), prouve l'estimation (3.5) du théorème.

Nous allons en déduire une version à croissance du théorème d'approximation de Bernstein-Walsh-Siciak.

THÉORÈME 3.2. Soit $K$ un compact localement non pluripolaire de $X$. Alors pour tout $\theta>1$ et $r_{1}>r_{0}:=\sup _{K} \exp l_{K}^{*}$, il existe une constante $c\left(r_{1}, \theta\right)=c>0$ telle que

$$
\begin{aligned}
& \varepsilon_{l}(f ; K) \leq c(1+r)^{d+n-1} r^{-l}\|f\|_{\Omega_{r \theta}}, \\
& \forall r \geq r_{1}, \quad \forall f \in \mathcal{O}\left(\bar{\Omega}_{r \theta}\right), \forall l \in \mathbb{N},
\end{aligned}
$$

où d est un entier qui ne dépend que de $X$.

Démonstration. Appliquons le théorème 3.1 avec $V=l_{K}^{*}$ : on a alors $D_{r}=\Omega_{r}$. Soit $\theta>1, \varrho \geq s>r_{2}>r_{1}$ et $f \in \mathcal{O}\left(\bar{\Omega}_{\varrho \theta}\right)$. D'après le théorème 3.1 , on a alors

$$
\int_{\Omega_{s}}\left|f-Q_{l}\right|^{2}(1+\tau)^{-d_{1}-2} \beta_{n}<c(\theta)(s / \varrho)^{2 l}\left(1+\varrho^{2}\right)^{d_{2}+n-1}\|f\|_{\bar{\Omega}_{\ell \theta}}^{2},
$$


où $Q_{l}$ est une fonction holomorphe vérifiant l'estimation (3.4) du théorème 3.1 .

En utilisant une version quantitative du théorème des fonctions implicites au voisinage de chaque point de $X$ pour se ramener à l'inégalité de la moyenne sur $\mathbb{C}^{n}$, on peut convertir les estimations $L^{2}(3.4)$ et (3.19) en estimations uniformes sur $X$ (voir [De], lemme 15.7). On en déduit alors qu'il existe un entier $N>0$ ne dépendant que de $d_{1}$ et une constante $c_{1}>0$ tels que

$$
\left|Q_{l}\right|^{2} \leq c_{1}(1+\tau)^{l+N}, \quad \forall l \in \mathbb{N} .
$$

De la même façon on montre qu'il existe $c_{2}=c\left(r_{1}, s\right)>0$ telle que

$$
\left\|f-Q_{l}\right\|_{\bar{\Omega}_{r_{2}}}^{2} \leq c_{2} \int_{\Omega_{s}}\left|f-Q_{l}\right|^{2}(1+\tau)^{-d_{1}-2} \beta_{n} .
$$

Il est bien connu que l'estimation (3.20) implique que $Q_{l} \in A(X)$ (voir $[\mathrm{R}-\mathrm{W}])$ et donc $Q_{l} \in A_{l+N}(X)$ pour tout $l$. On en déduit facilement compte tenu de (3.21) qu'il existe une constante $c_{3}=c\left(r_{1}, s, \theta\right)$ et un entier $d$ ne dépendant que de $X$ tels que

$$
\varepsilon_{l}\left(f ; \bar{\Omega}_{r_{2}}\right) \leq c_{3}(s / \varrho)^{l}(1+\varrho)^{d+n-1}\|f\|_{\bar{\Omega}_{\varrho \theta}}, \quad \forall l \in \mathbb{N} .
$$

D'après le corollaire 2.2 il en résulte qu'il existe une constante $c_{4}=c\left(r_{1}, s, \theta\right)$ telle que

$$
\varepsilon_{l}(f ; K) \leq c_{4}\left(\frac{s}{r_{1} \varrho}\right)^{l}(1+\varrho)^{d+n-1}\|f\|_{\bar{\Omega}_{\varrho \theta}}, \quad \forall l \in \mathbb{N} .
$$

En choisissant $s=r_{1} \theta$ et $r_{2}$ tel que $r_{1}<r_{2}<r_{1} \theta$ et en posant $\varrho=r \theta$ dans l'estimation (3.23), on en déduit l'estimation (3.18) du théorème avec $\theta^{2}$ au lieu de $\theta$. Comme $\theta>1$ est arbitrairement fixé, cela achève la démonstration du théorème.

R e m ar que. Si $X=\mathbb{C}^{n}$, le théorème est valable avec $d=2$, car $d_{2}=0$ et $N=2$.

Le théorème 3.1 a une autre conséquence concernant l'approximation sur un compact pluripolaire complet de $X$ (voir [Si,3]). Rappelons qu'un fermé $Y \subset X$ est dit pluripolaire complet s'il existe $u$ p.s.h. sur $X$ telle que $Y=\{x \in X: u(x)=-\infty\}$.

On peut montrer que la fonction $u$ peut être choisie dans la classe $\mathcal{L}(X)$ (voir $[\mathrm{B}-\mathrm{T}])$.

On a alors le résultat suivant :

THÉORÈme 3.3. Soit $K$ un compact pluripolaire complet de $X$. Alors pour tout $f \in \mathcal{O}(K)$ on a $\lim _{l \rightarrow \infty} \varepsilon_{l}(f ; K)^{1 / l}=0$, la limite étant uniforme en $f$ sur toute partie bornée de $\mathcal{O}(K)$. 
Démonstration. On peut facilement montrer qu'il existe $V \in \mathcal{L}(X)$ telle que $V^{-1}(-\infty)=K$ et $V$ continue sur $X-K([\mathrm{Ze}, 3])$. En tronquant $V$ en dehors d'un voisinage compact de $K$, on peut supposer que $V$ est exhaustive sur $X$ et vérifie l'estimation (3.1) en dehors d'un compact. Comme le comportement à l'infini n'importe pas dans ce théorème, la minoration dans (3.1) est inutile.

En reprenant la méthode utilisée dans la démonstration du théorème 3.2 , on obtient l'estimation suivante :

$$
\varepsilon_{l}(f ; K) \leq c(r, \theta)(s / r)^{l}\|f\|_{\bar{D}_{r \theta}}, \quad \forall l \in \mathbb{N}, \forall f \in \mathcal{O}\left(\bar{D}_{r \theta}\right),
$$

pour $0<s<r$ et $\theta>1$, où $D_{r}:=\{x \in X: V(x)<\log r\}$.

Soit $U$ un voisinage ouvert de $K$. On peut alors trouver $r>0$ tel que $D_{r} \Subset U$. L'estimation précédente implique alors que $\lim \sup _{l \rightarrow \infty} \varepsilon_{l}(f ; K)^{1 / l}$ $\leq s / r$ uniformément pour $f$ appartenant à une partie bornée de $\mathcal{O}(U)$. Comme $s>0$ est arbitraire, le théorème en résulte.

4. Meilleure approximation polynomiale et croissance des fonctions entières. Nous allons appliquer le théorème 3.2 pour mettre en évidence le lien précis qui existe entre la rapidité de l'approximation polynomiale d'une fonction entière et sa croissance.

Soit $X$ une sous-variété algébrique lisse de $\mathbb{C}^{\nu}$, de dimension pure $n, K$ un compact localement non pluripolaire de $X$ et $l_{K}^{*}$ sa fonction de Green pluricomplexe définie au paragraphe 2 .

Pour $r>r_{0}:=\sup _{K} \exp l_{K}^{*}$, posons

$$
\Omega_{r}:=\left\{x \in X: l_{K}^{*}(x)<\log r\right\} .
$$

Soit $f \in \mathcal{O}(X)$. On définit l'ordre de $f$ par la formule

$$
\varrho(f):=\limsup _{r \rightarrow \infty} \frac{\log ^{+} \log \|f\|_{\bar{\Omega}_{r}}}{\log r} .
$$

Cette définition ne dépend pas du compact $K$.

Si $f \in \mathcal{O}(X)$ est d'ordre fini $\varrho>0$, le nombre

$$
\sigma_{K}(f):=\limsup _{r \rightarrow \infty} \frac{\log \|f\|_{\bar{\Omega}_{r}}}{r^{\varrho}}
$$

dépend de $K$ et s'appelle le $K$-type de $f$ sur $X$.

Nous allons maintenant utiliser le théorème 3.2 pour établir le résultat suivant qui généralise celui que nous avons obtenu dans [Ze,1].

ThÉORÈme 4.1. Soit $K$ un compact localement non pluripolaire de $X$ et $\varrho>0$. 
1) Si f est une fonction holomorphe sur $X$ d'ordre $\varrho$ et de K-type fini $\sigma$, alors

$$
\limsup _{l \rightarrow \infty} \frac{l}{e \varrho} \varepsilon_{l}(f ; K)^{\varrho / l}=\sigma .
$$

2) Inversement, sif est une fonction continue sur $K$ telle que

$$
\sigma^{\prime}:=\limsup _{l \rightarrow \infty} \frac{l}{e \varrho} \varepsilon_{l}(f ; K)^{\varrho / l}<\infty,
$$

alors $f$ se prolonge en une fonction holomorphe sur $X$ d'ordre $\varrho$ et de K-type fini $\sigma$ donné par $\sigma=\sigma^{\prime}$.

Démonstration. 1) Supposons que $f$ soit une fonction entière sur $X$ d'ordre $\varrho>0$ et de $K$-type fini $\sigma$. Pour estimer la suite $\varepsilon_{j}(f ; K)$, utilisons le théorème 3.2. Alors pour tout $\theta>1$, il existe $C_{\theta}>0$ telle que

$$
\varepsilon_{l}(f ; K) \leq c_{\theta}(1+r)^{d} r^{-l}\|f\|_{\bar{\Omega}_{r \theta}}, \quad \forall r>r_{0}, \forall l \in \mathbb{N} .
$$

Par définition (voir (4.3)) pour tout $\sigma_{1}>\sigma$, il existe $c_{1}>0$ telle que

$$
(1+r)^{d}\|f\|_{\bar{\Omega}_{r}} \leq c_{1} e^{\sigma_{1} r^{\varrho}}, \quad \forall r>r_{0} .
$$

Il résulte alors de (4.4) et (4.5) que l'on a

$$
\varepsilon_{l}(f ; K) \leq c^{\prime}(\theta) r^{-l} e^{\sigma_{1} r^{\varrho}} .
$$

Par conséquent, on en déduit que

$$
\text { (4.7) } \frac{l}{e \varrho} \varepsilon_{l}(f ; K)^{\varrho / l} \leq c^{\prime}(\theta)^{\varrho / l} \frac{l}{e \varrho} r^{-\varrho} \exp \left(\frac{1}{l} \varrho \sigma_{1} r^{\varrho}\right), \quad \forall r>r_{0}, \quad \forall l \geq 1 \text {. }
$$

Pour $l$ fixé assez grand, le second membre de (4.7) atteint son minimum pour $r$ vérifiant $\varrho r^{\varrho} / l=1 / \sigma_{1}$. En reportant cette valeur dans (4.7), nous obtenons l'inégalité suivante :

$$
\frac{l}{e \varrho} \varepsilon_{l}(f ; K)^{\varrho / l} \leq c^{\prime}(\theta)^{\varrho / l} \sigma_{1}, \quad \forall l \geq l_{0},
$$

où $l_{0}$ est un entier assez grand pour que $\left(l_{0} /\left(\varrho \sigma_{1}\right)\right)^{1 / \varrho}>r_{0}$.

En passant à la limite dans (4.8), on en déduit que $\sigma^{\prime} \leq \sigma_{1}$, pour tout $\sigma_{1}>\sigma$. D'où $\sigma^{\prime} \leq \sigma$.

2) Supposons maintenant que $f$ est une fonction continue sur $K$ et que $\sigma^{\prime}<\infty$. Nous allons montrer que $f$ se prolonge en une fonction entière sur $X$ d'ordre $\varrho$ et de $K$-type $\sigma \leq \sigma^{\prime}$. En effet, soit $\beta>\sigma^{\prime}$; il existe alors un entier $l_{0}>1$ tel que

$$
\varepsilon_{l}(f ; K) \leq\left(\beta e \varrho l^{-1}\right)^{l / \varrho}, \quad \forall l \geq l_{0} .
$$

On sait qu'il existe $P_{l} \in A_{l}(X)$ tel que $\varepsilon_{l}(f ; K)=\left\|f-P_{l}\right\|_{K}$ pour chaque $l$. Il en résulte d'après $(4.9)$ que $\left(P_{l}\right)$ converge uniformément vers $f$ 
sur $K$. Autrement dit,

$$
f=P_{l_{0}}+\sum_{l=l_{0}}^{\infty}\left(P_{l+1}-P_{l}\right) \quad \text { sur } K .
$$

D'après l'inégalité de Bernstein-Walsh sur $X$ (voir $\S 2)$, on a

$$
\left\|P_{l+1}-P_{l}\right\|_{\bar{\Omega}_{r}} \leq\left\|P_{l+1}-P_{l}\right\|_{K} r^{l+1}, \quad \forall r>r_{0}, \forall l \geq 1 .
$$

Comme $\left\|P_{l+1}-P_{l}\right\|_{K} \leq\left\|f-P_{l+1}\right\|_{K}+\left\|f-P_{l}\right\|_{K} \leq 2 \varepsilon_{l+1}(f ; K)$, il en résulte compte tenu de (4.9) et (4.10) que l'on a

(4.11) $\quad\left\|P_{l+1}-P_{l}\right\|_{\bar{\Omega}_{r}} \leq 2 r\left(\beta e \varrho r^{\varrho} l^{-1}\right)^{l / \varrho}, \quad \forall r>r_{0}, \forall l \geq r_{0}$.

L'inégalité (4.11) implique que la série $P_{l_{0}}+\sum_{l>l_{0}}\left(P_{l+1}-P_{l}\right)$ converge uniformément sur tout compact de $X$ vers une fonction holomorphe sur $X$ qui coïncide avec $f$ sur $K$. Nous noterons encore $f$ ce plongement : $f=$ $P_{l_{0}}+\sum_{l=l_{0}}^{\infty}\left(P_{l+1}-P_{l}\right)$ sur $X$. Il résulte alors de (4.11) que l'on a

$$
\|f\|_{\bar{\Omega}_{r}} \leq\left\|P_{l_{0}}\right\|_{\bar{\Omega}_{r}}+2 r \sum_{l=1}^{\infty}\left(\beta \varrho e r^{\varrho} l^{-1}\right)^{l / \varrho}, \quad \forall r>r_{0} .
$$

Pour $r$ fixé $>r_{0}$, le maximum du terme général de la série du second membre de (4.12) est atteint pour $l=\beta \varrho r^{\varrho}$ et vaut $e^{\beta r^{\varrho}}$. Posons $l_{2}=$ $\left[2^{\varrho} e \varrho \beta r^{\varrho}\right]$ et découpons la somme de la série en deux termes :

$$
S=\sum_{l=1}^{l_{2}} C_{l}+\sum_{l=l_{2}+1}^{\infty} C_{l}, \quad \text { où } \quad C_{l}=\left(\beta e \varrho r^{\varrho} l^{-1}\right)^{l / \varrho} .
$$

D'après le choix de $l_{2}$, on a

$$
\sum_{l=1}^{l_{2}} C_{l} \leq l^{2} e^{\beta r^{e}} \quad \text { et } \quad \sum_{l=l_{2}+1}^{\infty} C_{l} \leq \sum_{l=1}^{\infty} \frac{1}{2^{l}}<1 .
$$

Il en résulte compte tenu de (4.12) que l'on a

$$
\|f\|_{\bar{\Omega}_{r}} \leq C r^{l_{0}}+(2 r)^{\varrho+1} \varrho \varrho \beta e^{\beta r^{\varrho}}+2 r, \quad \forall r>r_{0} .
$$

On en déduit facilement que $f$ est d'ordre $\varrho$ et de type $\sigma \leq \beta$ pour tout $\beta>$ $\sigma^{\prime}$ et donc $\sigma \leq \sigma^{\prime}$. Compte tenu de la première partie de la démonstration, on en déduit que $\sigma=\sigma^{\prime}$.

Re marque. Ce résultat généralise le théorème que nous avons obtenu dans $[\mathrm{Ze}, 1]$. Lorsque $X=\mathbb{C}$, on obtient un théorème dû à Winiarski ([Wi,1]) et lorsque $K$ est un produit de compacts de $\mathbb{C}$, on obtient un résultat de Nguyen T. V. $([\mathrm{Ng}])$.

5. Extension holomorphe avec contrôle de la croissance. Soit $X$ une sous-variété algébrique (non singulière) de $\mathbb{C}^{\nu}$ de dimension pure $n$. Il 
est bien connu que toute fonction holomorphe sur $X$ se prolonge en une fonction entière sur $\mathbb{C}^{\nu}$.

Björk a montré qu'il existe un entier $b=b(X)$ tel que toute fonction $f$ holomorphe sur $X$ et vérifiant $|f(x)| \leq c(1+|x|)^{l}$ pour tout $x \in X$ se prolonge en un polynôme sur $\mathbb{C}^{\nu}$ de degré $\leq l+b$ ([Bj]; voir [No] pour une démonstration plus élémentaire).

Djakov et Mityagin ont construit un opérateur linéaire continu naturel d'extension de $\mathcal{O}(X)$ dans $\mathcal{O}\left(\mathbb{C}^{\nu}\right)$ qui préserve certains types de croissance $([\mathrm{D}-\mathrm{M}])$.

Il existe dans la littérature de nombreux résultats concernant le problème d'extension avec contrôle de la croissance et il est difficile de citer tous les résultats connus. Nous renvoyons à $[R, 2]$ pour une bibliographie plus complète.

Ici nous nous intéressons à l'extension des fonctions holomorphes avec un contrôle quantitatif précis du type de croissance. Nous allons donner une généralisation du théorème de Björk au cas des fonctions entières (voir théorème 5.1).

Soit $U_{r}$ le polydisque de $\mathbb{C}^{\nu}$ défini par

$$
U_{r}=\left\{z \in \mathbb{C}^{\nu}:|z|=\sup _{1 \leq i \leq \nu}\left|z_{i}\right|<r\right\}, \quad r>0 .
$$

Soit $F \in \mathcal{O}\left(\mathbb{C}^{\nu}\right)$ et $\varrho(r)$ un ordre précisé pour l'ordre $\varrho>0$ ([L-G]). On définit le type de $F$ pour l'ordre précisé $\varrho(r)$ par la formule suivante :

$$
\sigma(F)=\sigma(F ; \varrho)=\varlimsup_{r \rightarrow \infty} \frac{\log \|F\|_{\bar{U}_{r}}}{r \varrho(r)} .
$$

De la même façon, si $f \in \mathcal{O}(X)$ on définit le type de $f$ sur $X$ pour l'ordre précisé $\varrho(r)$ par la formule

$$
\sigma(f)=\varlimsup_{r \rightarrow \infty} \frac{\log \|f\|_{X \cap \bar{U}_{r}}}{r^{\varrho(r)}} .
$$

ThÉORÈmE 5.1. Il existe un opérateur linéaire continu d'extension $E$ : $\mathcal{O}(X) \rightarrow \mathcal{O}\left(\mathbb{C}^{\nu}\right)$ vérifiant les estimations suivantes :

$\exists R>0, \forall \theta>1, \exists C_{\theta}>0$ tels que

$$
\|E(f)\|_{\bar{U}_{r}} \leq C_{\theta}(1+r)^{d}\|f\|_{X \cap \bar{U}_{r \theta}}, \quad \forall f \in \mathcal{O}(X), \forall r \geq R
$$

où $d$ est un entier ne dépendant que de $X$.

Corollaire 5.2. Soit $\varrho(r)$ un ordre précisé associé à l'ordre $\varrho$. Alors pour tout $f \in \mathcal{O}(X)$ de type $\sigma$ pour l'ordre précisé $\varrho(r)$, le prolongement $E(f)$ est une fonction entière sur $\mathbb{C}^{\nu}$ de type $\sigma$ pour le même ordre précisé $\varrho(r)$.

Avant de procéder à la démonstration de ces résultats, rappelons la construction de Djakov et Mityagin de l'opérateur d'extension $E$. 
Notons $\leq$ l'ordre défini sur $\mathbb{N}^{\nu}$ de la façon suivante : $\alpha \leq \beta$ si et seulement si $|\alpha|<|\beta|$ ou bien $|\alpha|=|\beta|$ et il existe $k, 1 \leq k \leq \nu$, tel que $\alpha_{k}<\beta_{k}$ et $\alpha_{j}=\beta_{j}$ pour $j=1, \ldots, k-1($ si $k>2)$.

Suivant Djakov et Mityagin $([\mathrm{D}-\mathrm{M}])$ posons

$$
S=\left\{\alpha \in \mathbb{N}^{\nu}: z^{\alpha} \notin\left[z^{\beta} ; \beta \leq \alpha\right]+I\right\},
$$

où $I=I(X)$ est l'idéal polynomial de $X$ et $\left[z^{\beta} ; \beta \leq \alpha\right]$ est le sous-espace vectoriel de $\mathbb{C}\left[z_{1}, \ldots, z_{\nu}\right]$ engendré par $z^{\beta}, \beta \leq \alpha$. On définit alors les espaces de Fréchet suivants :

$$
\begin{aligned}
& \mathcal{O}_{S}\left(\mathbb{C}^{\nu}\right):=\left\{f \in \mathcal{O}\left(\mathbb{C}^{\nu}\right): f=\sum_{\alpha \in \mathbb{N}^{\nu}} c_{\alpha} z^{\alpha}, c_{\alpha}=0 \forall \alpha \notin S\right\}, \\
& \mathcal{O}_{S}\left(U_{r}\right):=\left\{f \in \mathcal{O}\left(U_{r}\right): f=\sum_{\alpha \in \mathbb{N}^{\nu}} c_{\alpha} z^{\alpha}, c_{\alpha}=0 \forall \alpha \notin S\right\} .
\end{aligned}
$$

D'après Djakov et Mityagin ([D-M]) l'opérateur de restriction

$$
\mathcal{O}_{S}\left(\mathbb{C}^{\nu}\right) \rightarrow \mathcal{O}(X), \quad F \mapsto f=F \mid X,
$$

est un isomorphisme d'espaces de Fréchet dont l'isomorphisme inverse défini par

$$
E: \mathcal{O}(X) \rightarrow \mathcal{O}_{S}\left(\mathbb{C}^{\nu}\right) \subset \mathcal{O}\left(\mathbb{C}^{\nu}\right), \quad f \mapsto \tilde{f}
$$

est un opérateur linéaire continu d'extension "naturel" associé à l'ordre $\leq$ choisi sur $\mathbb{N}^{\nu}$. C'est cet opérateur qui intervient dans le théorème 5.1.

On peut expliciter cet opérateur : d'après ce qui précède, si on note $e_{\alpha}(x)=x^{\alpha}$ pour $x \in X, \alpha \in S$, alors tout $f \in \mathcal{O}(X)$ s'écrit de façon unique

$$
f=\sum_{\alpha \in S} a_{\alpha} e_{\alpha} \quad \operatorname{dans} \mathcal{O}(X) .
$$

Le prolongement naturel $\widetilde{f}=E(f)$ s'écrit alors

$$
\widetilde{f}=\sum_{\alpha \in S} a_{\alpha} z^{\alpha} \quad \operatorname{dans} \mathcal{O}\left(\mathbb{C}^{\nu}\right) .
$$

De plus, si $R>0$ est assez grand pour que $U_{R}$ rencontre chaque composante irréductible de $X$, l'isomorphisme (5.6) induit les isomorphismes suivants :

$$
\mathcal{O}_{S}\left(U_{r}\right) \rightarrow \mathcal{O}\left(X \cap U_{r}\right), \quad r \geq R .
$$

Démonstration du théorème 5.1. Posons $K=X \cap \bar{U}_{R}$. Il est clair, d'après le choix de $R$, que $K$ est localement non pluripolaire dans $X$. On peut donc appliquer le théorème 3.2.

Fixons $\delta>1$; il existe alors une constante $C_{\delta}>0$ telle que

$$
\varepsilon_{l}(f ; K) \leq C_{\delta}(1+r)^{d} r^{-l}\|f\|_{\bar{\Omega}_{r \delta}}, \quad \forall l \in \mathbb{N}, \forall r>r_{1},
$$

où $\Omega_{r \delta}=\Omega(K ; r \delta)$. 
Fixons $f \in \mathcal{O}(X)$. On sait (voir [Ze,2]) qu'il existe pour chaque $l \in \mathbb{N}$ un "polynôme" $Q_{l} \in A_{l}(X)$ tel que $\varepsilon_{l}(f ; K)=\left\|f-Q_{l}\right\|_{K}$. D'après Dyakov et Mityagin $([\mathrm{D}-\mathrm{M}])$ (voir aussi $[\mathrm{Bj}]$ et $[\mathrm{No}]$ ), il existe un entier $b=b(X) \geq 0$ tel que pour tout $Q \in A_{l}(X)$, le prolongement $\widetilde{Q}$ soit un polynôme de degré $\leq l+b$. Posons $H_{0}=Q_{1}$ et $H_{l}:=Q_{l}-Q_{l-1}$ pour $l \geq 1$. On a alors

$$
\left\|H_{0}\right\|_{K} \leq 2\|f\|_{K} \quad \text { et } \quad\left\|H_{l}\right\|_{K} \leq 2 \varepsilon_{l-1}(f ; K), \quad \forall l \in \mathbb{N}^{*} .
$$

Il en résulte alors, compte tenu de (5.9), que l'on a

$$
\left\|H_{l}\right\|_{K} \leq 2 C_{\delta}(1+r)^{d} r^{-l+1}\|f\|_{\bar{\Omega}_{r \delta}}, \quad \forall r>1, \forall l \geq 0 .
$$

D'après la continuité de l'isomorphisme (5.8), pour tout $\eta \in] 0,1[$, il existe une constante $C_{1}=C_{1}(\eta)>0$ telle que

$$
\left\|\widetilde{H}_{l}\right\|_{\bar{U}_{\eta R}} \leq C_{1}\left\|H_{l}\right\|_{X \cap \bar{U}_{R}}, \quad \forall l \geq 0 .
$$

D'après (5.10) et (5.11) et le fait que $K=X \cap \bar{U}_{R}$, il existe une constante $C_{2}=C_{2}(\eta, \delta)>0$ telle que

$$
\left\|\widetilde{H}_{l}\right\|_{\bar{U}_{\eta R}} \leq C_{2}(1+r)^{d+1} r^{-l}\|f\|_{\bar{\Omega}_{r \delta}}, \quad \forall l \geq 0, \forall r \geq r_{1} .
$$

L'inégalité de Bernstein-Walsh pour les polynômes sur $\mathbb{C}^{\nu}$ implique à partir de (5.12) l'estimation suivante :

$$
\begin{aligned}
\left\|\widetilde{H}_{l}\right\|_{\bar{U}_{s R}} \leq C_{2}(1+r)^{d+b+1}\left(\frac{s}{\eta r}\right)^{l+d}\|f\|_{\bar{\Omega}_{r \delta}}, & \\
& \forall l \geq 0, \forall r \geq r_{1}, \forall s>1 .
\end{aligned}
$$

Soit $\theta>1$; on choisit $\delta=\sqrt{\theta}, r=s \sqrt{\theta}$, avec $s>r_{1}$ et $\eta<1$ tel que $\eta \sqrt{\theta}>1$. On a alors

$$
\sum_{l=0}^{\infty}\left\|\widetilde{H}_{j}\right\|_{\bar{U}_{s R}} \leq C_{3}(1+s)^{d+b+1}\|f\|_{\bar{\Omega}_{s \theta}}, \quad \forall s>1
$$

où $C_{3}=C_{3}(\theta)$ est indépendante de $s$ et $f$. La série $\sum_{l=0}^{\infty} \widetilde{H}_{l}$ converge donc vers une fonction entière $g$ sur $\mathbb{C}^{\nu}$ telle que $g=f$ sur $K$. Comme $K$ est localement non pluripolaire dans $X$, on en déduit que $g=f$ sur $X$. Autrement dit, $g=\widetilde{f}$ est le prolongement de $f$ défini par l'opérateur (5.7), et la formule (5.14) implique

$$
\|\widetilde{f}\|_{\bar{U}_{s R}} \leq C_{3}(1+s)^{d+b+1}\|f\|_{\bar{\Omega}_{s \theta}}, \quad \forall s>r_{1} .
$$

Comme $K=X \cap \bar{U}_{R} \subset \bar{U}_{R}$, les fonctions de Green associées dans $\mathbb{C}^{\nu}$ vérifient les inégalités $L_{\bar{U}_{R}} \leq L_{K}$ sur $\mathbb{C}^{\nu}$. On sait de plus que $L_{K} \mid X=l_{K}$ et donc $\Omega_{r} \subset X \cap U_{r R}$ pour tout $r>1$, ce qui d'après (5.15) prouve l'inégalité (5.3) du théorème. 
Démonstration du corollaire 5.2. Si $f \in \mathcal{O}(X)$ est de type $\sigma$ pour l'ordre précisé $\varrho(r)$, on a

$$
\sigma=\limsup _{r \rightarrow \infty} \frac{\log \|f\|_{X \cap \bar{U}_{r}}}{r \varrho(r)} .
$$

D'après le théorème 4.1 , si $F=\widetilde{f}$ est l'extension de $f$ par l'opérateur $E$, on a

$$
\limsup _{r \rightarrow \infty} \frac{\log \|F\|_{\bar{U}_{r}}}{r^{\varrho(r)}} \leq \limsup _{r \rightarrow \infty} \frac{\log \|f\|_{X \cap \bar{U}_{r \theta}}}{r^{\varrho(r)}}, \quad \forall \theta>1 .
$$

Comme $\lim _{r \rightarrow \infty}(\theta r)^{\varrho(\theta r)} / r^{\varrho(r)}=\theta^{\varrho}$ (voir [L-G]), on obtient

$$
\sigma(F) \leq \theta^{\varrho} \sigma, \quad \forall \theta>1 .
$$

D'où $\sigma(F) \leq \sigma$. Comme évidemment $\sigma(f) \leq \sigma(F)$, on obtient $\sigma(F)=\sigma=$ $\sigma(f)$.

Remarques. 1) Des résultats du type précédent ont été obtenus par Ronkin dans le cas des hypersurfaces algébriques de $\mathbb{C}^{\nu}([\mathrm{R}, 1])$.

2) Soit $Y$ un cône algébrique de $\mathbb{C}^{\nu}$. Alors il est facile de prouver que si $K$ est un compact disqué de $Y$, on a

$$
\varepsilon_{l}(f ; K) \leq \frac{r}{r-1} \cdot \frac{\|f\|_{\bar{\Omega}_{r}}}{r^{l+1}}, \quad \forall f \in \mathcal{O}(Y), \forall l \in \mathbb{N}, \forall r>1 .
$$

On en déduit aisément que le prolongement naturel $\widetilde{f}$ de $f$ à $\mathbb{C}^{\nu}$ vérifie l'estimation

$$
\|\widetilde{f}\|_{\bar{U}_{r}} \leq C(\theta) \frac{r}{r-1}\|\widetilde{f}\|_{Y \cap U_{r \theta}}, \quad \forall r>1 .
$$

Il est naturel de conjecturer que les estimations des théorèmes 3.2 et 5.1 restent valables pour toute variété algébrique avec ou sans singularités.

\section{Bibliographie}

[B-T] E. Bedford and B. A. Taylor, Plurisubharmonic functions with logarithmic singularities, Ann. Inst. Fourier (Grenoble) 38 (4) (1988), 133-171.

[Be] S. Bernstein, Sur l'ordre de la meilleure approximation polynomiale des fonctions continues, Bruxelles, 1912.

[Bj] J.-E. Björk, On extensions of holomorphic functions satisfying a polynomial growth condition on algebraic varieties in $\mathbb{C}^{n}$, Ann. Inst. Fourier (Grenoble) 24 (4) (1974), 157-165.

[De] J.-P. Demailly, Mesures de Monge-Ampère et caractérisation géométrique des variétés algébriques affines, Mém. Soc. Math. France 19 (1985).

[D-M] P. B. Djakov and B. S. Mityagin, The structure of polynomial ideals in the algebra of entire functions, preprint of the Institute of Mathematics, Polish Academy of Sciences, no. 123, 1977.

[H] L. Hörmander, An Introduction to Complex Analysis in Several Variables, Van Nostrand, Princeton, 1973. 
[L-G] P. Lelong and L. Gruman, Entire Functions of Several Variables, Springer, 1986.

[Ng] T. V. Nguyen, Croissance et meilleure approximation polynomiale des fonctions entières, Ann. Polon. Math. 24 (1972), 325-333.

[No] K. J. Nowak, The extension of holomorphic functions of polynomial growth on algebraic sets in $\mathbb{C}^{n}$, Osnabrücker Schriften zur Math., 1984.

[R,1] L. L. Ronkin, Continuation with estimates of holomorphic functions on sets of zeros of polynomials, Teor. Funktsiu Funktsional. Anal. i Prilozhen. 128, 36 (1981), 89-103.

$[\mathrm{R}, 2] \quad-$, Entire functions, in: Encyclopedia of Math. Sci., Several Complex Variables III, Springer, 1986, 1-30.

[R-W] K. Rusek and T. Winiarski, Criteria for regularity of holomorphic mappings, Bull. Acad. Polon. Sci. Sér. Sci. Math. 28 (1980), 471-475.

[Sa] A. Sadullaev, An estimate for polynomials on analytic sets, Math. USSR-Izv. 20 (1980), 493-502.

[Si,1] J. Siciak, On some extermal functions and their applications in the theory of analytic functions of several complex variables, Trans. Amer. Math. Soc. 105 (1962), 322-357.

[Si,2] - Extremal plurisubharmonic functions in $\mathbb{C}^{n}$, Ann. Polon. Math. 39 (1981), $175-211$.

[Si,3] —, Approximation by transcendental polynomials, ibid. 46 (1985), 299-309.

[Sk] H. Skoda, Morphismes surjectifs et fibrés linéaires semi-positifs, dans : Séminaire P. Lelong-H. Skoda (Analyse), Lecture Notes in Math. 694, Springer, 1978.

[Wa] J. Walsh, Interpolation and Approximation by Rational Functions, Boston, 1960.

[Wi,1] T. Winiarski, Approximation and interpolation of entire functions, Ann. Polon. Math. 23 (1970), 259-273.

[Wi,2] —, Application of approximation and interpolation methods to the examination of entire functions of $n$ variables, ibid. 28 (1973), 98-121.

[Za] V. P. Zakharyuta, Extremal plurisubharmonic functions, orthogonal polynomials and the Bernstein-Walsh theorem for analytic functions of several complex variables, Ann. Polon. Math. 33 (1976), 137-148 (in Russian).

[Ze,1] A. Zeriahi, Meilleure approximation polynomiale et croissance des fonctions holomorphes sur une variété algébrique affine, Ann. Inst. Fourier (Grenoble) 37 (2) (1987), 79-104.

[Ze,2] - Fonction de Green pluricomplexe à pôle à l'infini sur un espace de Stein parabolique et applications, Math. Scand. 69 (1991), 89-126.

$[\mathrm{Ze}, 3] \quad$ - Ensembles pluripolaires exceptionnels pour la croissance partielle des fonctions holomorphes, Ann. Polon. Math. 50 (1989), 81-91.

LABORATOIRE D'ANALYSE

UNIVERSITÉ PAUL SABATIER

118, ROUTE DE NARBONNE

31062 TOULOUSE CEDEX, FRANCE 